\title{
Object Oriented Modeling and Control Design for Power Electronics Half-Bridge Converter using Modelica
}

\author{
Giuseppe Laera $^{1} \quad$ Luigi Vanfretti ${ }^{1} \quad$ Kyle Thomas $^{2} \quad$ Matthew Gardner $^{2}$ \\ ${ }^{1}$ ECSE, Rensselaer Polytechnic Institute, Troy (NY), \{laerag, vanfrl $\}$ erpi.edu \\ ${ }^{2}$ Dominion Energy, Richmond (VA), \{kyle.thomas, matthew.gardner $\}$ @dominionenergy .com
}

\begin{abstract}
In this paper the focus is on a particular type of converters that is the two-level VSC (DC/AC Voltage Source Converter). In this category the averaged model and the switching model of an half-bridge converter are considered. The half-bridge is a building block for multiphase and multilevel converters.

The implementation of the two models of half-bridge converter using Modelica language is described with the structure of the package developed in Dymola. Different control strategies are introduced showing different behavior of the models in the simulations. The goals of this paper are several. First of all the modeling choice was to use Modelica for this type of work, that is traditionally carried out with domain specific tools, to show that it is possible to perform implementation and studies in the same field where traditional commercial softwares have been commonly and extensively used, with additional benefits that the language provides. In addition to that, this paper shows that the control design studies for an half-bridge converter, typically performed using averaged value models, can result in a set of control parameters values that are not successfully applicable to switching models of the same power electronic device.

Keywords: VSC converters, half-bridge, averaged model, switching model, STATCOM, Modelica, Dymola, PI control, lead compensator, lag compensator, resonant control
\end{abstract}

\section{Introduction}

\subsection{Motivations}

With the enhancements of power semiconductors the application of power electronics has been extended from traditional domestic and industrial applications to electric power systems. The power-electronic converters for power systems are used especially for power compensation and power filtering. Such converters consist of a power circuit realized through different configurations of switches and passive components coupled with a control system (Yazdani and Iravani, 2010).

In the past, the applications of power-electronic converter systems in electric power systems were limited to the HVDC (High Voltage Direct Current) transmission systems and static reactive power compensators like SVC (Static Var Compensator). With time the application ar- eas for generation, transmission and distribution of electric energy have been extended for several reasons. The main incentives are represented by:

- Continuosly growing development of power electronics technology for electric power systems

- Development of signal processing and control strategies

- Issues with power line congestions

- Growing of energy consumption leading to the utilization of the existent electric infrastructure at its technical limits (stability issues)

- Increasing penetration of renewable energy sources due to the economic feasibility and to environmental concerns

The use of power-electronic converters in power systems is also motivated by the need to improve the efficiency and reliability of the existent electric infrastructure for integrating large scale renewable energy sources and storage systems.

The main applications of power-electronic converters in power systems are:

- Active filters: they synthesize and inject specific components of current or voltage into the grid to improve the power quality (Rashid, 2017)

- Compensation: the aim is to improve the power transfer capability of the lines, the voltage stability, the power quality. In this category we have the STATCOM (Static Synchronous Compensator). The function of the STATCOM is to act as power compensator by injecting or absorbing reactive power from the grids (see Figure 1).

- Power conditioning: the idea is to allow for a power exchange between two electrical systems under control to meet specific requirements like frequency, voltage magnitude, etc. (Maza-Ortega et al., 2017)

This paper focuses on the implementation and design of a simple power electronic DC/AC converter, the halfbridge. The first modeling choice is to use Modelica for 


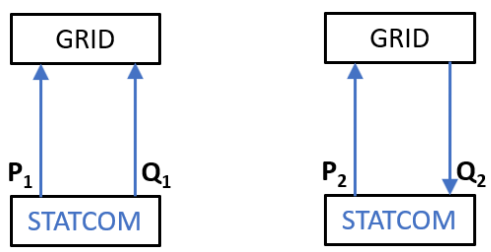

Figure 1. Behavior of the STATCOM as compensator (reactive power Q1 injection and reactive power Q2 absorption).

this type of work that is traditionally carried out with domain specific tools. In contrast with the current practice, the use of a modern object-oriented equation based programming language like Modelica allows to show that it is possible to perform implementation and studies in the same field where traditional commercial softwares have been commonly and extensively used, with additional benefits that the language provides. The models used in this paper have been chosen on purpose from the literature (referenced in the bibliography) to show that it is possible to obtain comparable results with this alternative tool instead of the classical PSCAD, etc.; and so that a reader from the power engineering community can quickly realize the value of the Modelica approach.

\subsection{Previous Works}

Modeling of power electronic converters in Modelica has been described in previous studies like (Haumer and Kral, 2011). It is a very informative work and it provides a very thorough description for an active front end converter with PWM (three phase AC/DC converter), however, from the authors point of view it requires that the reader is already quite familiar with Modelica. The paper also deals with an example more appropriate for engineers in the area of machine drives. The aspect of the synchronization of the control of the inverter to the grid voltage, discussed in (Haumer and Kral, 2011), would require the implementation of a PLL (Phase-Locked Loop) component that is part of the on-going work of the authors for a three-phase full STATCOM model. The main objective of this paper is to use the application illustrated as the starting point for the implementation of more complex power electronics models and it is focusing more on the differences of a couple of output current control strategies and tuning depending on the considered model of the half-bridge converter if averaged or switching.

In power system analysis studies, different levels of modeling detail are used to represent the converter switches. This is because when simulating a large number of switches, for example in modular multilevel converters, even ideal switches will lead to very large simulation time. Hence, different modeling approaches to represent each module (i.e. switches) are used (Saad et al., 2016). In this paper the switching behavior of the switches is considered ideal. This means that they can be turned off or on instantaneously. In reality a switch, combination of a transistor and its anti-parallel diode, cannot be turned on and off instantaneously. Those processes require a so called dead time or blanking time during which the signal to the gate of the switch is set to zero to reach the complete on or off condition. In other words the switching devices have finite turn-on and turn-off delays (Zammit et al., 2016). This is necessary because if we consider the leg of the halfbridge converter, as in this paper, if one switch has not yet completed the turn-off process and, at the same time, the other switch is turned-on then a shoot-through failure (short-circuit current through the leg) can happen damaging the converter (De Doncker et al., 2010). The dead time introduces a non-linearity which causes distortion in the output voltage and current (Zammit et al., 2016).

Another example of half bridge converter implementation using Modelica is given in (Winter et al., 2015) where a DC/DC converter, extensively used in the automotive industry, is considered. An averaged model of the converter is taken into account and the study covers aspects like converter losses in addition to a validation versus a SPICE model.

In (Olenmark and Sloth, 2014) an investigation about the implementation of different control strategies for power electronic converters has been performed in Modelica with the software tool Dymola, the same used in this paper. However, a different tuning method for PI controllers is implemented in this paper. It is the lambda method described in (McMillan et al., 1999), (Pruna et al., 2017) and usually applied in several industrial processes.

\subsection{Contributions}

In this paper a simple power electronic system has been chosen as the basis for more complex power electronics devices such as a three-phase STATCOM. One of the goals of the authors is to illustrate to the power engineering community the value of adopting Modelica, and consequently our approach attempts to make emphasis on how Modelica can be used for typical control design tasks considering that the reader is not so familiar with Modelica. For example, the simple use of records serves to illustrate how to use them for control design and not only provide parameter data. Because the community in North America that uses Modelica is still developing, the authors believe that this paper can provide a good instructional value to the readers, especially since we are making the models available on GitHub ${ }^{1}$.

The modeling approach was based on using the Modelica Standard Library to build all the components with diagram blocks similarly to a tool like PSCAD and Simulink. One of the advantages of using Modelica and the Modelica Standard Library is that the developed models can work in different software environments like OpenModelica, Dymola, Wolfram SystemModeler, SimulationX, etc. without the need to load external third party libraries. This

\footnotetext{
${ }^{1}$ https://github.com/ALSETLab/Modeling-Control-Design-PowerElectronics-Half-Bridge-Converter-Modelica
} 
is impossible with the current tools used by the power engineering community.

\section{Half-bridge converter applications and modeling}

In this section the authors present the typical modeling approaches used to represent the half-bridge converter, which will be implemented in the next section. This converter can be seen as basic building block used to model more complex power electronics systems. As an example consider a STATCOM connected to the AC grid as shown in Figure 2. The whole system includes the DC/AC converter, the control system, the transformer and the heat exchanger. The DC/AC converter and the control system in this STATCOM are built from a basic half-bridge topology. Hence, in this paper, the main goal is to derive an implementation of the basic topology needed to build a full STATCOM converter (see Figure 3).

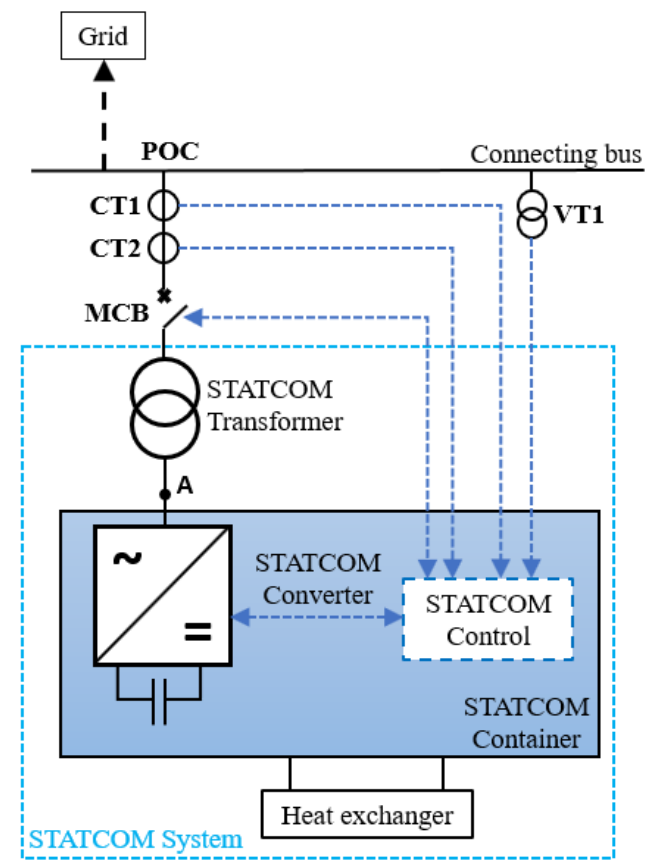

Figure 2. Connection of the STATCOM to the AC grid.

In other words, the system considered in this project is the first step in developing a 3-phase, three-wire, two-level VSC converter that can represent a STATCOM. The halfbridge is the basic building block needed to implement either three phase VSC's through parallel combination (see Figure 3) or multimodule VSC converters through parallel/series combination.

The two models of the half-bridge converter of this paper are represented in Figure 4 and Figure 5. They also include a resistance in series to each switch (current generator in Figure 4 and transistor in Figure 5) representing the non ideal effect of the on-state resistance of the switches.

The two models have been implemented using Model-

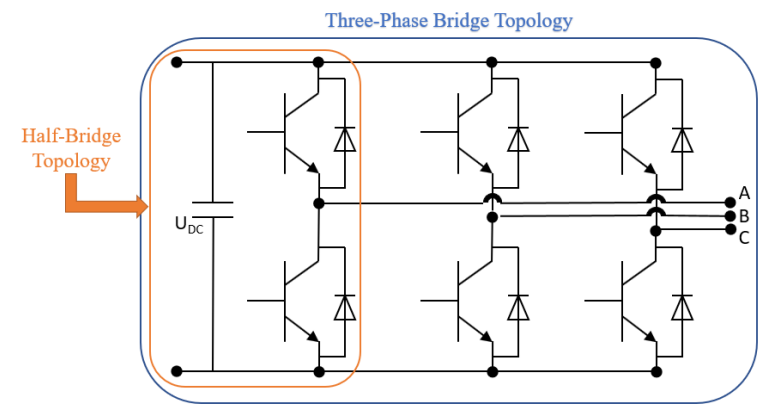

Figure 3. Half-bridge converter as building block of a three phase VSC converter.

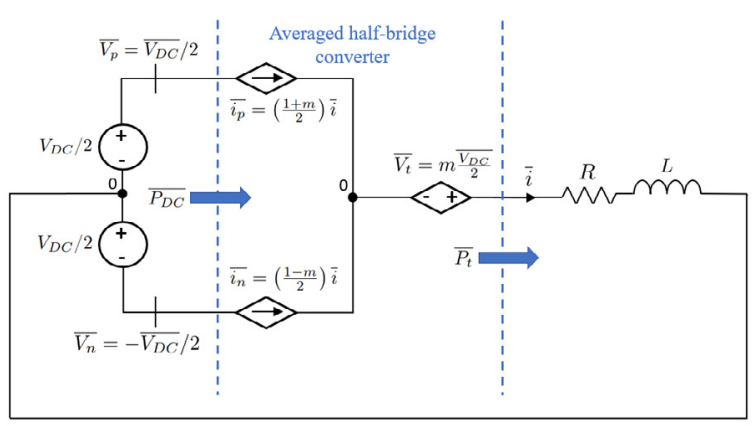

Figure 4. Averaged equivalent circuit of the half-bridge converter.

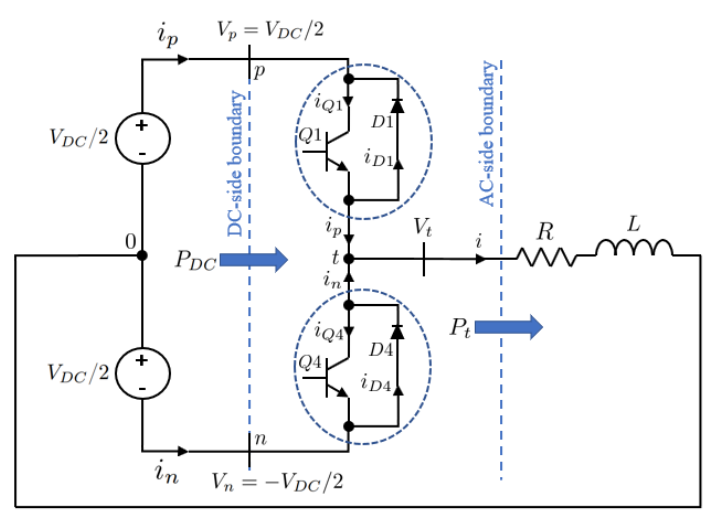

Figure 5. Switching equivalent circuit of the half-bridge converter. 
ica language in the software environment Dymola. Modelica is an open-source object oriented language that is suitable for multidomain modeling of physical systems (Vanfretti et al., 2013).

If we look at Figure 4, the dynamics of the AC current $i$, that is the output of the system, can be described by Equation (1).

$$
L \frac{d i}{d t}+\left(R+r_{o n}\right) i=V_{t}
$$

In Equation (1) $R$ and $L$ are the parameters of the AC system to which the converter is connected to and $r_{o n}$ is the on-state resistance of each switch of the converter. A switch is represented in this case by an equivalent current source (see Figure 4) that is not ideal because of the parameter $r_{o n}$. For the analysis of this system $r_{o n}$ is lumped with the resistance $R$. From Equation (1) we can consider the AC current $i$ as state variable and $V_{t}=m V_{D C} / 2$ as our control input since it can be changed by varying $m$, the modulating signal.

A block diagram of the system represented by Equation (1) is given in Figure 6 (Yazdani and Iravani, 2010).

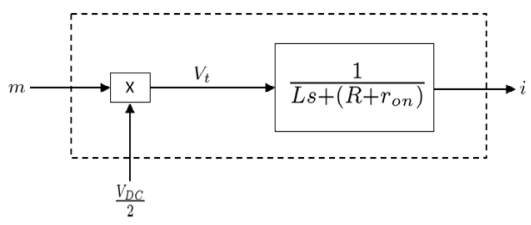

Figure 6. Block diagram of the $\mathrm{AC}$ side of the half-bridge converter.

\section{Modelica model design and imple- mentation}

The objective of this paper is to implement the two models of the half-bridge converter in Modelica. The structure of the package created to model all the components and systems is given in Figure 7. The different averaged and switching models with and without controls are listed at the top of the package HalfBridgeConverter. The first two models of the list are the switching and averaged models without controls, the second couple of models represents those with a PI controller and the third couple of models represents those with a modified control strategy. A record package is used for tuning the PI controller through the lambda method. Given the input parameters of the system in the Plant record, the calculations of the PI parameters are performed by the formulas in the PI_Lambda record and the results are stored into the PI_par record used to set the values of the PI controller parameters. The package Components contains all the components used in the models listed at the top of the HalfBridgeConverter package. The components are created by using only the Modelica Standard Library.

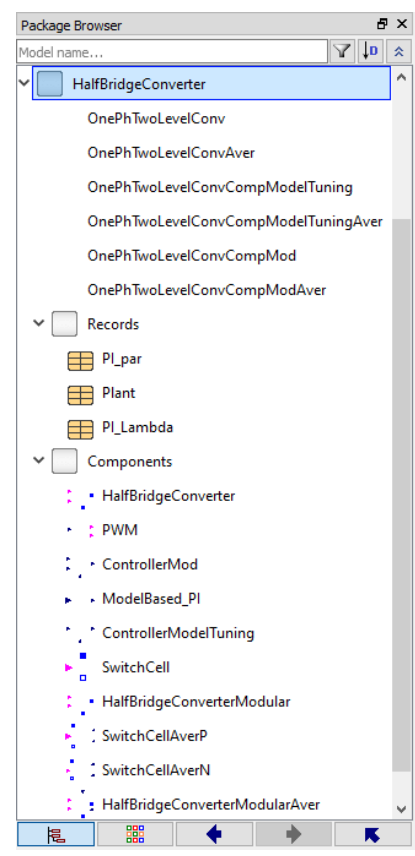

Figure 7. Modelica package for the models of this paper.

Another task of this paper regards the regulation of the output current $i$ of the open-loop system in Figure 6 to a specified reference signal.

\subsection{Modelica implementation}

The first step consisted of modeling the system in the two versions without any control. The averaged model of the half-bridge converter in Figure 4 is represented in Figure 8 .

The switching model of the half-bridge converter in Figure 5 is represented in Figure 9.

Each switch encapsulates another block diagram (see Figure 10 and Figure 11).

It should be noticed that for the averaged model the gates and their signals in Figure 8 and Figure 10 are not in use but they are already implemented for the switching model of the half-bridge converter in Figure 5. This will make easy to change the type of converter in the model of the whole system by simply changing the class of that component using the Modelica feature of replaceable.

The whole systems are then modeled as in Figure 12 and in Figure 13. The same systems with a PI controller are given in Figure 14 and in Figure 15. The output current ripple of the switching models in Figure 13 and Figure 15 can be reduced by introducing a capacitor in parallel to the series of resistance and inductance on the AC side of the converter.

The block of the controller in Figure 14 and Figure 15 is represented in Figure 16 and it includes the PI compensator in Figure 17.

Finally the systems with a more elaborated control strategy still look like as in Figure 14 and Figure 15 but in this case the controller block is represented in Figure 


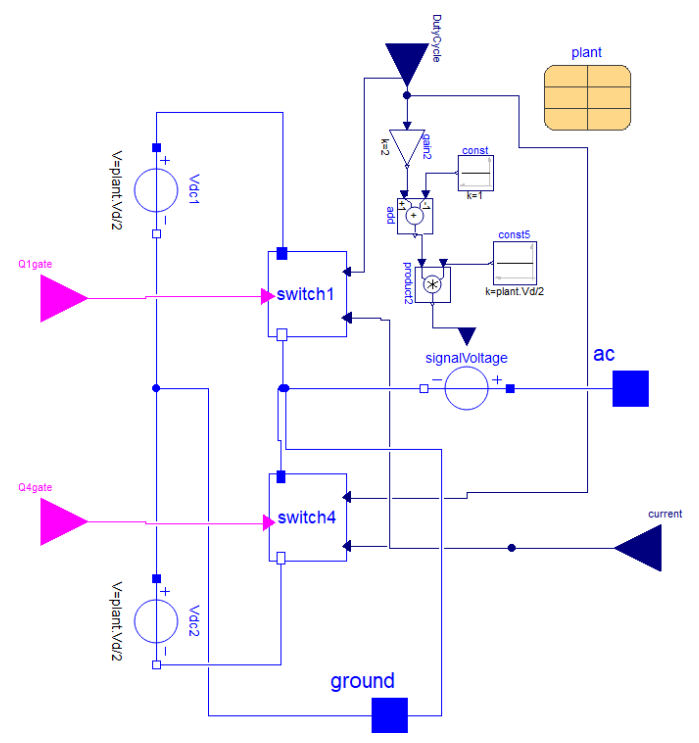

Figure 8. Block diagram of the averaged model of the halfbridge converter.

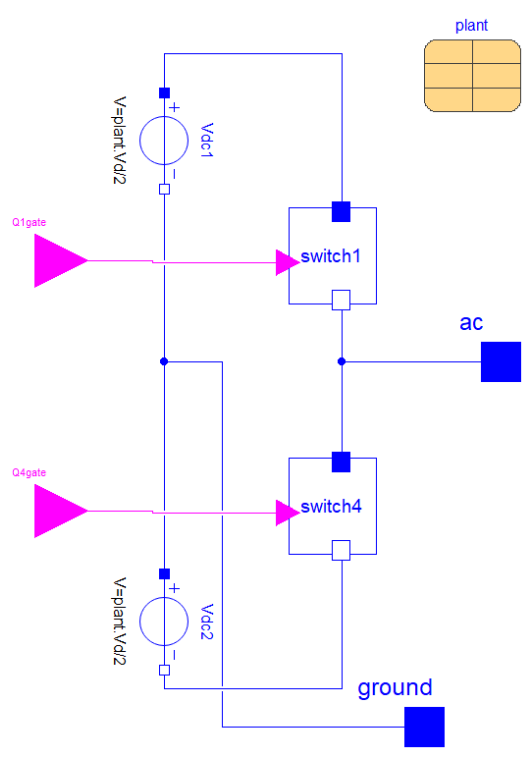

Figure 9. Block diagram of the switching model of the halfbridge converter.

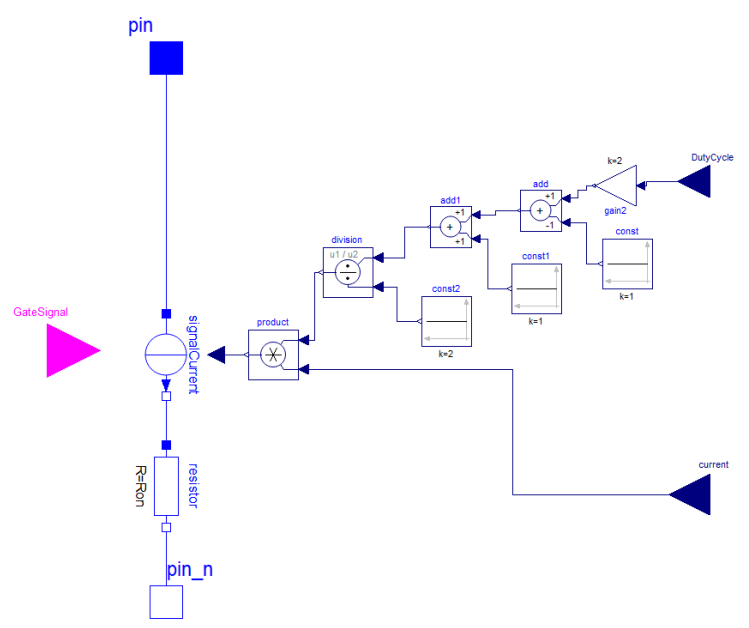

Figure 10. Block diagram of switchl in Figure 8.

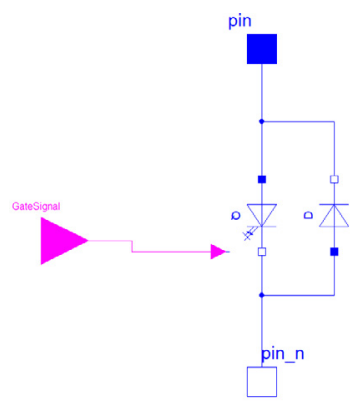

Figure 11. Block diagram of switchl in Figure 9.

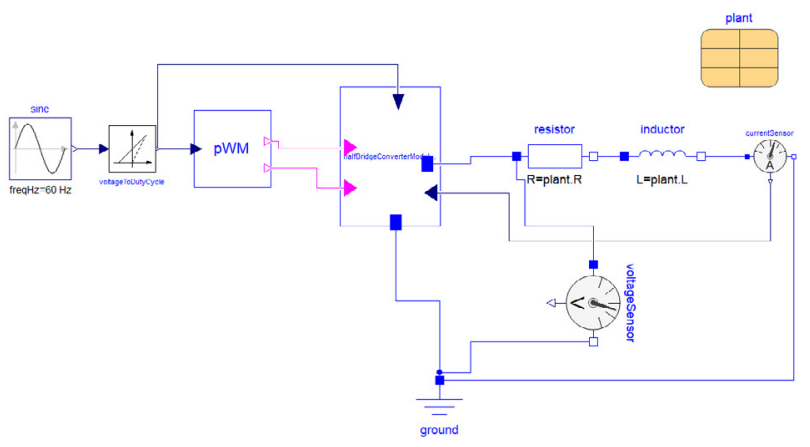

Figure 12. Block diagram of the system with the averaged model of the half-bridge converter without any control.

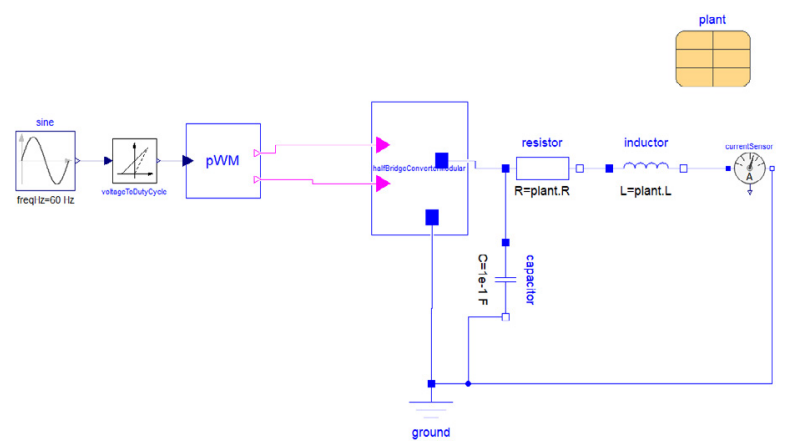

Figure 13. Block diagram of the system with the switching model of the half-bridge converter without any control. 
18.

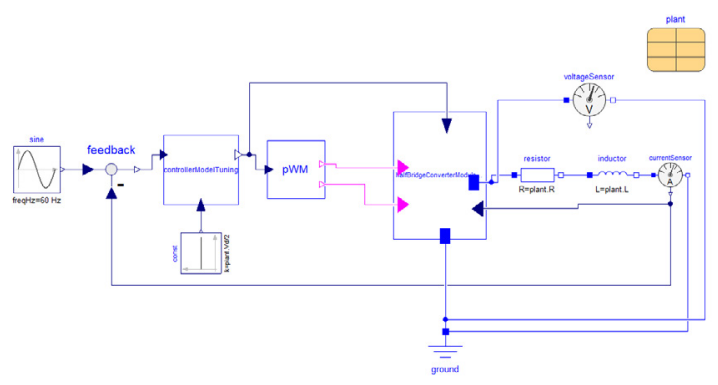

Figure 14. Block diagram of the system with the averaged model of the half-bridge converter with PI control.

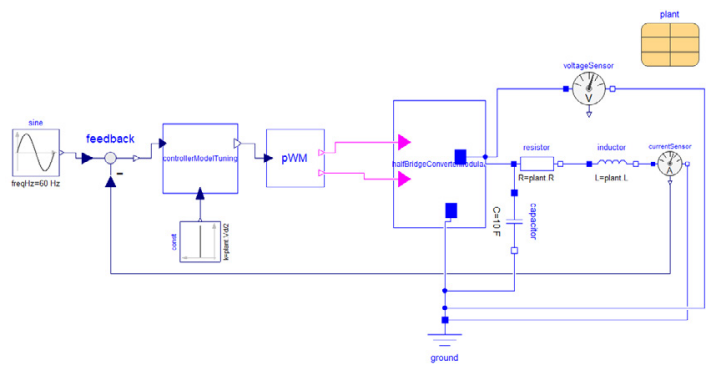

Figure 15. Block diagram of the system with the switching model of the half-bridge converter with PI control.

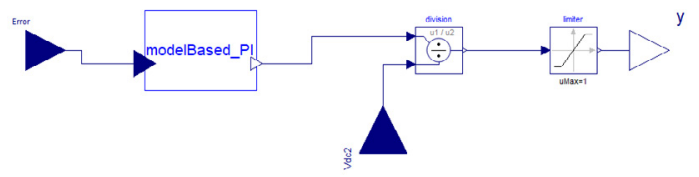

Figure 16. Block diagram of the controller in Figure 14 and Figure 15.

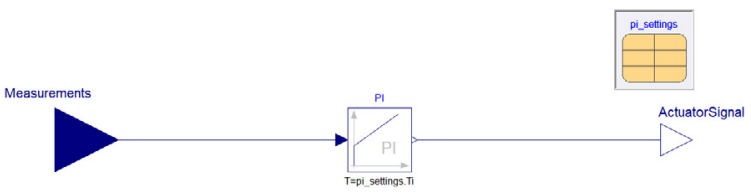

Figure 17. Block diagram of the PI compensator contained in the controller block of Figure 16.

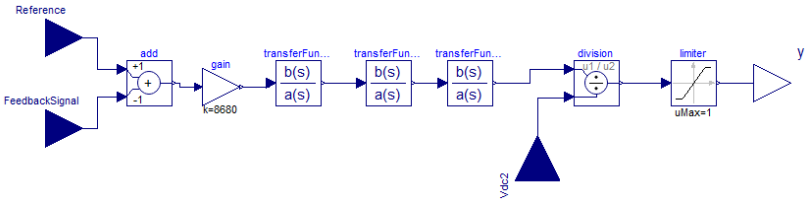

Figure 18. Block diagram of the controller with modified strategy for both the averaged and switching model.

In Figure 18 there is a gain and three blocks of transfer functions that have been added for this new control strategy. The three transfer functions correspond to a lead compensator, a lag compensator and a resonant control. This modification of the controller has been introduced because with only a PI controller it is not possible to track a sinusoidal reference waveform without error but only a step reference signal (Yazdani and Iravani, 2010).

\subsection{Control design of the PI for the averaged model of the half-bridge converter}

The control of the output current can be obtained by creating a closed-loop including a controller that takes as input an error signal generated by the difference between the output current $i$ and a reference current $i_{\text {ref }}$. The block diagram of the described closed-loop system is given in Figure 19.

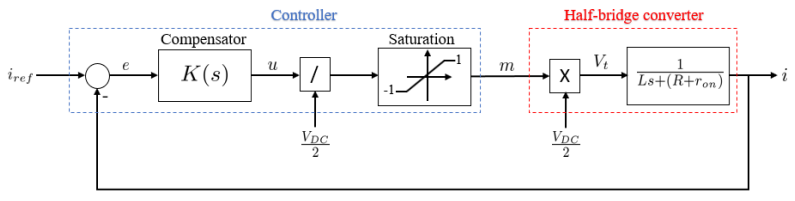

Figure 19. Block diagram of the closed-loop system with the half-bridge converter and a controller.

From Figure 19, the compensator $K(s)$ takes as input the error signal $e$ and gives as output the signal $u$ that is divided by $V_{D C} / 2$ to compensate for the voltage gain of the converter since $V_{t}=m V_{D C} / 2$. In addition to that, the modulating signal $m$ must satisfy $|m| \leq 1$ so the Saturation block has been introduced in the block diagram.

Depending on the reference signal and the performance to match different compensators may be used. If the reference signal $i_{r e f}$ is a step function then a proportionalintegral (PI) compensator can be considered for control purposes. Its generic descriptive expression is:

$$
K(s)=\frac{k_{p} s+k_{i}}{s}
$$

From Equation (2) and Figure 19 the open-loop transfer function of the system can be derived:

$$
G_{\text {open }}(s)=K(s) G(s)=\left(\frac{k_{p}}{L s}\right)\left(\frac{s+\frac{k_{i}}{k_{p}}}{s+\frac{R+r_{\text {on }}}{L}}\right)
$$


From Figure 6 it is possible to see that the open-loop system has a stable pole at $p=-\left(R+r_{o n}\right) / L$. In general, considering the typical values for $R, r_{o n}$ and $L$, the pole $p$ is quite close to the origin giving a slow natural response. So to improve the open-loop frequency response the pole $p$ can be cancelled by the zero of the PI compensator. Then, from Equation (3), it is possible to choose for the compensator $k_{i} / k_{p}=\left(R+r_{o n}\right) / L$ and $k_{p} / L=1 / \tau_{i}$ where $\tau_{i}$ is the desired time constant of the closed-loop system. With the previous choices the transfer function of the closed-loop system becomes:

$$
G_{\text {closed }}(s)=\frac{K(s) G(s)}{1+K(s) G(s)}=\frac{i}{i_{\text {ref }}}=\frac{1}{\tau_{i} s+1}
$$

The time constant $\tau_{i}$ should be small enough to have a fast response of the current control. But it has also to be considered that $1 / \tau_{i}$ should be smaller than the switching frequency of the half-bridge converter. Considering the mentioned aspects $\tau_{i}$ is usually in the range $0.5-5 \mathrm{~ms}$ and it can vary depending on the specific application of the converter and its switching frequency (Yazdani and Iravani, 2010).

\section{Simulations}

The analysis of results can start by considering as reference input of the half-bridge converter a step function with a step of amplitude $50 \mathrm{~A}$ applied at $0.1 \mathrm{~s}$. From Figure 12 we can just replace the sinusoidal source with a step block that is used to calculate the duty cycle $d$ necessary for the modulation factor $m(m=2 d-1)$ that is one of the inputs of the block halfBridgeConverter. The output of the halfBridgeConverter is connected to the AC system characterized by the components $R$ and $L$. The current sensor in series with $R$ and $L$ is used to measure the AC output current $i$ that is fed back as input of the halfBridgeConverter and that will be controlled in the following steps.

As application example, we can consider the following parameters values (Yazdani and Iravani, 2010):

- $L=690 \mu H$

- $R=5 m \Omega$

- $r_{\text {on }}=0.88 m \Omega$

- $\tau_{i}=5 m s$

Applying these parameters to the system in Figure 12 we can run a simulation and plot the step input and the output AC current of the system. The results are in Figure 20.

As we can see from Figure 20 the AC output current does not track the step input. The final value of the output AC current is much higher than the step input.

Then a PI compensator is introduced. From the parameters previously defined, we can derive $k_{p}=L / \tau_{i}=0.138$ and

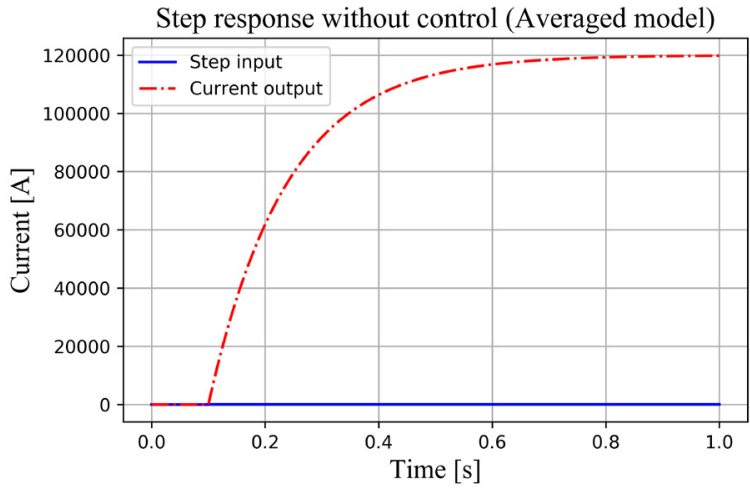

Figure 20. Plot of the step input (blue curve) and AC output current (red curve) of the system with averaged model without controls. Due to the difference of magnitudes between the two curves the step of $50 \mathrm{~A}$ cannot be seen in the figure.

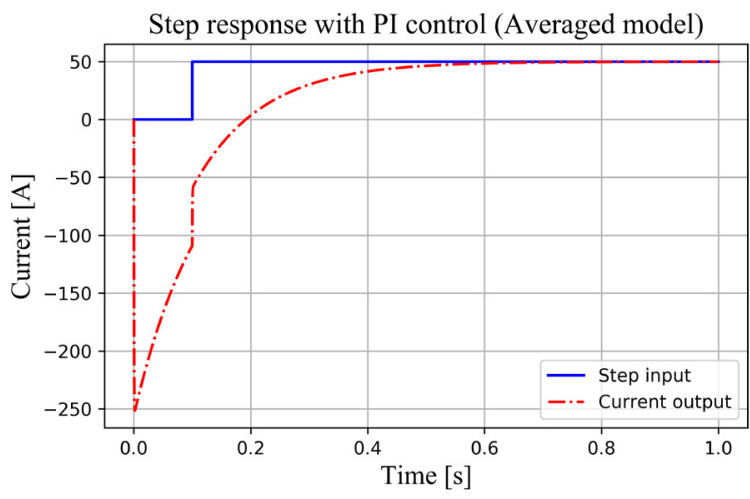

Figure 21. Plot of the step input (blue curve) and AC output current (red curve) of the system with averaged model with PI controller. 
$k_{i}=k_{p}\left(R+r_{\text {on }}\right) / L=1.176$. We can run a new simulation and the results are illustrated in Figure 21.

From Figure 21 we can see that now the system is able to settle at a final value corresponding to the step input value by using a PI controller. The initial negative overshoot of the current is due to the initial conditions of the system.

If we now consider the same conditions for the system with the switching model of the half-bridge converter, applying the same step without controls we get the results in Figure 23. Introducing the same PI control of the previous case we get the results in Figure 24. The value of the capacitance $\mathrm{C}$ at the output of the switching model of the converter can affect the output current ripple. There are also other requirements that need to be met, for example, the admissible voltage ripple on the load and a reasonable value of the capacitance C. An example is shown in Figure 22 where the switching model of the half-bridge converter without any control has been used. It shows the impact of the variation of the capacitance on the output current.

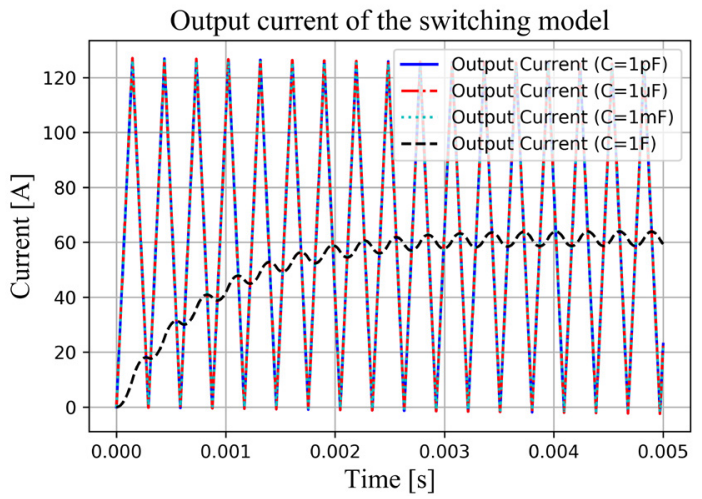

Figure 22. Plot of the AC output current of the system with switching model without controls for different values of the output capacitance C.

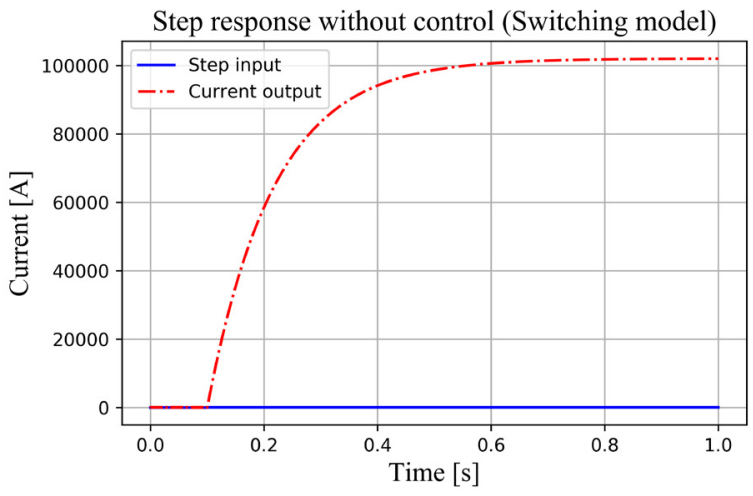

Figure 23. Plot of the step input (blue curve) and AC output current (red curve) of the system with switching model without controls. Due to the difference of magnitudes between the two curves the step of $50 \mathrm{~A}$ cannot be seen in the figure.

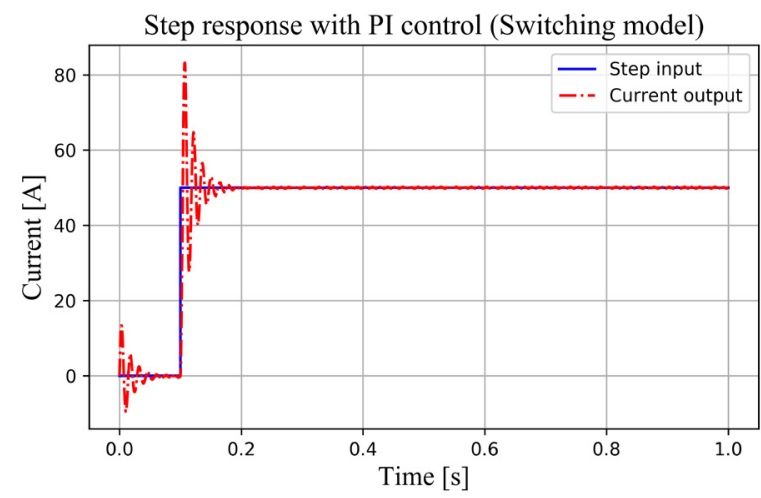

Figure 24. Plot of the step input (blue curve) and AC output current (red curve) of the system with switching model with PI controller.

From Figure 20 and Figure 23 we can see that without any control the output current is not able to track the step reference either with the averaged model or with the switching model. Introducing a PI controller, from Figure 21 and Figure 24, the results are better even if we have a large negative overshoot for the system with the averaged model of the half-bridge converter and some oscillations of the output current at the initialization of the system with the switching model when the step is applied.

The next test consists of changing the source of the reference signal with a sinusoid of amplitude 1000 and frequency $60 \mathrm{~Hz}$ starting at $t=0.07 \mathrm{~s}$. Running a simulation we get the results in Figure 25 for the system with the switching model without any control.

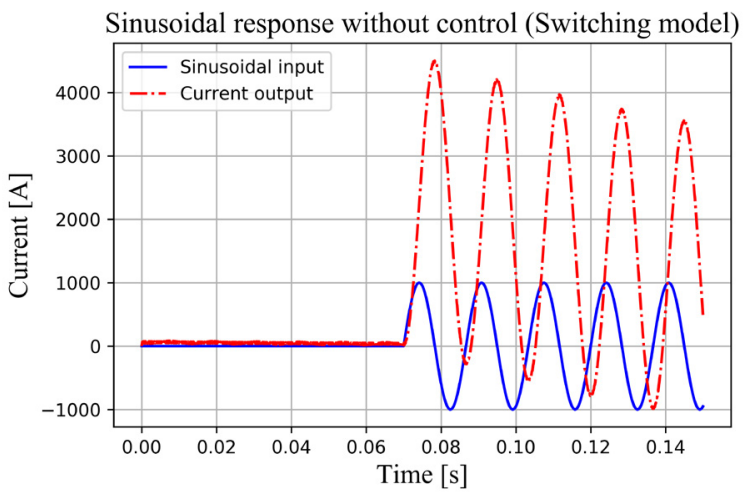

Figure 25. Plot of the sinusoidal input (blue curve) and AC output current (red curve) of the system with the switching model without controls.

For the system with the averaged model without any control we get the results in Figure 26.

From Figure 25 and Figure 26 the behavior of the systems with the switching model and the averaged model without any control looks very similar but not able to track the reference signal.

When introducing the same PI controller with the sinu- 


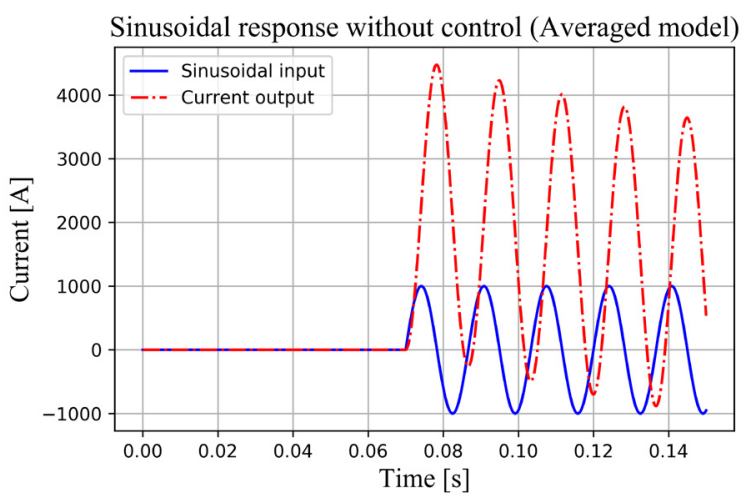

Figure 26. Plot of the sinusoidal input (blue curve) and AC output current (red curve) of the system with the averaged model without controls.

soidal reference signal we get the results in Figure 27 for the system with the switching model and the results in Figure 28 for the system with the averaged model.

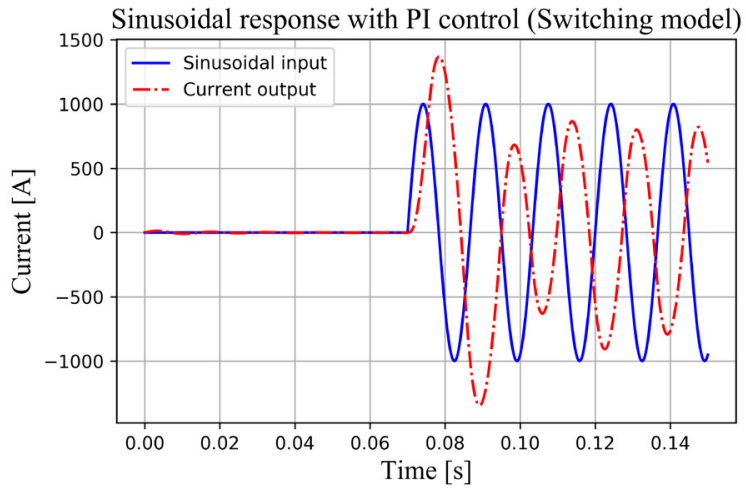

Figure 27. Plot of the sinusoidal input (blue curve) and AC output current (red curve) of the system with the switching model with PI controller.

From Figure 27 and Figure 28 we can see that the behavior of the system is better with the averaged model of the half-bridge converter than with the switching model. They both present errors in amplitude and phase compared to the reference but the averaged model looks like more aligned with the reference.

Then introducing a more elaborated control strategy described in 3.1 a new simulation with the sinusoidal reference can be performed. The results for the system with the switching model are in Figure 29 and for the system with the averaged model in Figure 30.

From Figure 29 and Figure 30 the behavior of the system with the switching model is better than the one with the averaged model since the tuning of the control parameters has been performed on the switching model.

In order to get a similar behavior of the system with the averaged model, like in Figure 31, the gain of the controller has been increased from a value of 8680 to 450000 .

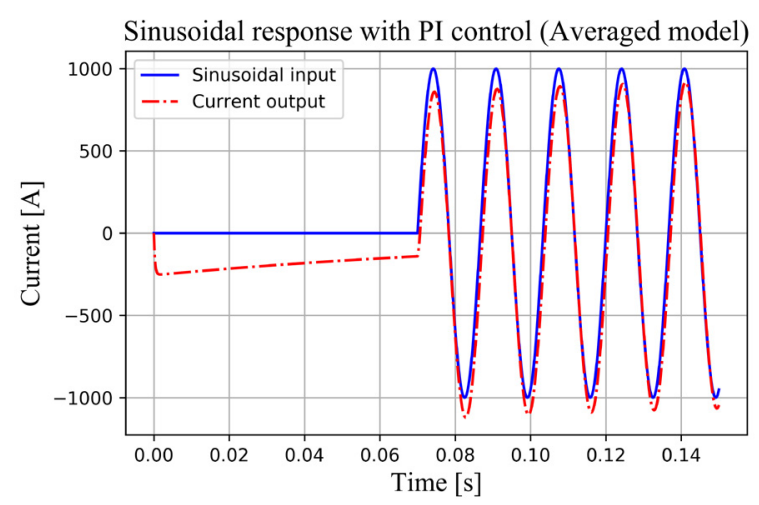

Figure 28. Plot of the sinusoidal input (blue curve) and AC output current (red curve) of the system with the averaged model with PI controller.

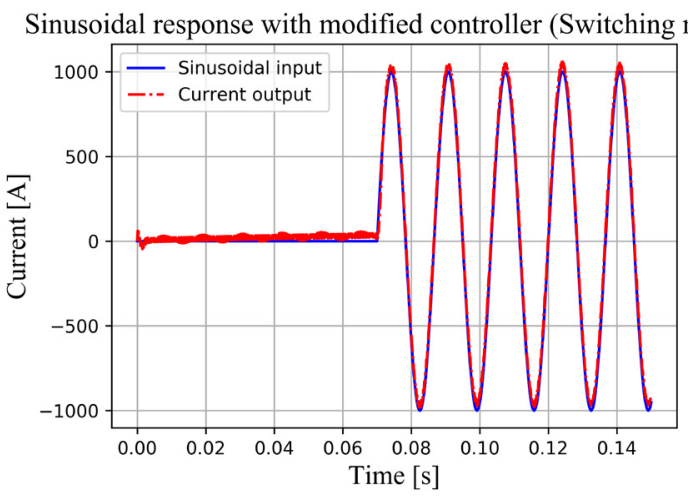

Figure 29. Plot of the sinusoidal input (blue curve) and AC output current (red curve) of the system with the switching model with modified controller.

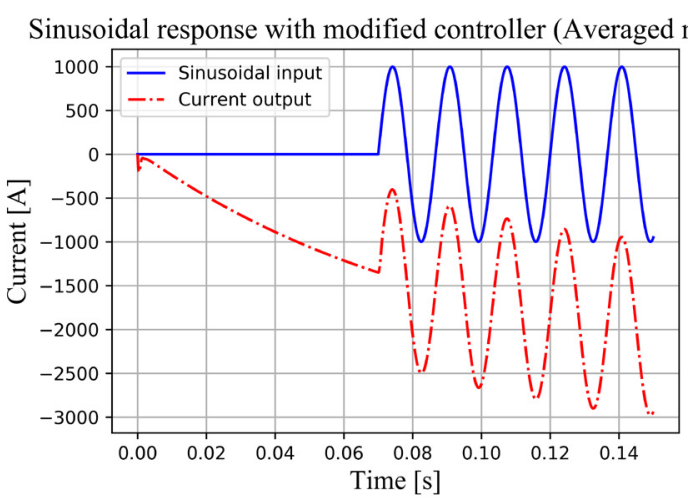

Figure 30. Plot of the sinusoidal input (blue curve) and $\mathrm{AC}$ output current (red curve) of the system with the averaged model with modified controller. 


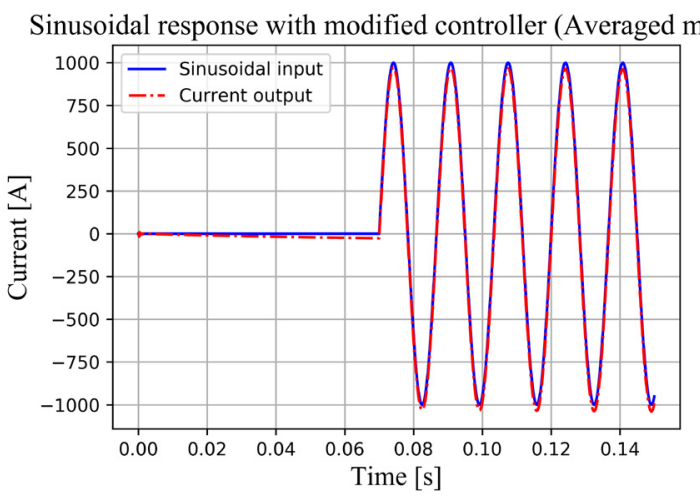

Figure 31. Plot of the sinusoidal input (blue curve) and AC output current (red curve) of the system with the averaged model with modified controller.

The voltage distortion due to voltage drop at grid's R-L due to non-sinusoidal currents of switching converter has not been shown because the focus of the paper is on the control of the output current of the half-bridge converter. There are also other aspects that can be analyzed starting from what has been presented like voltage distortion, THD content, other control strategies and so on. They can be studied in a future work.

\section{Further Work and Conclusions}

The modularity of blocks of components and records allows for an easy reuse in different models. This allowed to run simulations very quickly. One of the objectives of this paper is to communicate to the power system analysts the benefits of Modelica language features, such as the replaceable and redeclare features. In most power system tools, the user would have to set up different system models when using different representations. In future work, the authors plan to illustrate how we can use these features by changing from detailed component models, to the ideal switches, to the averaged value model. Simplifying model development and management.

The simulations show that the introduction of a feedback control improves the reference tracking. A specific combination of control parameters can work for a system with the switching model of the half-bridge converter but not for the one with the averaged model or viceversa. This will also be the case if we consider a more detailed or less detailed model of the switches, which will be illustrated in a future work. So for the tuning of the controls other tools can be considered for the estimation of their parameters. Other control strategies can be analyzed, for example, to reduce the initial overshoot of the output current of the system with the averaged model of the half-bridge or the initial oscillations of the system with the switching model. They represent an additional stress for the components of the converter.

The implementation of a three-phase converter will follow this work that is an initial step. So different control strategies can be taken into account for this more complex case.

\section{References}

Rik De Doncker, Duco WJ Pulle, and André Veltman. Advanced electrical drives: analysis, modeling, control. Springer Science \& Business Media, 2010.

Anton Haumer and Christian Kral. Modeling a mains connected pwm converter with voltage-oriented control. In Proceedings of the 8th International Modelica Conference; March 20th22nd; Technical Univeristy; Dresden; Germany, number 63, pages 388-397. Linköping University Electronic Press, 2011.

JM Maza-Ortega, E Acha, S García, and A Gomez-Exposito. Overview of power electronics technology and applications in power generation transmission and distribution. Journal of Modern Power Systems and Clean Energy, 5(4):499-514, 2017.

Gregory K McMillan, Douglas M Considine, et al. Process/industrial instruments and controls handbook, volume 7. McGraw Hill, 1999.

Andreas Olenmark and Jens Sloth. Flexible ac/dc grids in dymola/modelica-modeling and simulation of power electronic devices and grids. CODEN: LUTEDX/TEIE, 2014.

Edwin Pruna, Edison R Sasig, and Santiago Mullo. Pi and pid controller tuning tool based on the lambda method. In 2017 CHILEAN Conference on Electrical, Electronics Engineering, Information and Communication Technologies (CHILECON), pages 1-6. IEEE, 2017.

Muhammad H Rashid. Power electronics handbook. Butterworth-Heinemann, 2017

Hani Saad, Sébastien Dennetière, and Jean Mahseredjian. On modelling of mmc in emt-type program. In 2016 IEEE 17th Workshop on Control and Modeling for Power Electronics (COMPEL), pages 1-7. IEEE, 2016.

Luigi Vanfretti, Wei Li, Tetiana Bogodorova, and Patrick Panciatici. Unambiguous power system dynamic modeling and simulation using modelica tools. In 2013 IEEE Power \& Energy Society General Meeting, pages 1-5. IEEE, 2013.

Michael Winter, Sascha Moser, Stefan Schoenewolf, Julian Taube, and Hans-Georg Herzog. Average model of a synchronous half-bridge dc/dc converter considering losses and dynamics. In Proceedings of the 11th International Modelica Conference, Versailles, France, September 21-23, 2015, number 118, pages 479-484. Linköping University Electronic Press, 2015 .

Amirnaser Yazdani and Reza Iravani. Voltage-sourced converters in power systems, volume 34. Wiley Online Library, 2010.

Daniel Zammit, Cyril Spiteri Staines, and Maurice Apap. Compensation techniques for non-linearities in h-bridge inverters. Journal of Electrical Systems and Information Technology, 3 (3):361-376, 2016. 